\title{
In Vitro Antimicrobial Susceptibility of Mycobacterium massiliense Recovered from Wound Samples of Patients Submitted to Arthroscopic and Laparoscopic Surgeries
}

\author{
Alessandra Marques Cardoso, Ana Paula Junqueira-Kipnis, and André Kipnis \\ Departamento de Microbiologia, Imunologia, Parasitologia e Patologia, Instituto de Patologia Tropical e Saúde Pública, \\ Universidade Federal de Goiás, Rua Delenda Rezende Melo com $1^{a}$ Avenida, S/No , Setor Universitário, Goiânia, \\ 74605-050 Goiás, Brazil
}

Correspondence should be addressed to André Kipnis, andre.kipnis@gmail.com

Received 14 September 2010; Accepted 26 November 2010

Academic Editor: Kristi L. Harold

Copyright ( 2011 Alessandra Marques Cardoso et al. This is an open access article distributed under the Creative Commons Attribution License, which permits unrestricted use, distribution, and reproduction in any medium, provided the original work is properly cited.

\begin{abstract}
Testing of rapidly growing species of mycobacteria (RGM) against antibacterial agents has been shown to have some clinical utility. This work establishes the MICs of seven antimicrobial agents following the guidelines set forth by the Clinical and Laboratory Standards Institute (CLSI) against eighteen isolates of Mycobacterium massiliense recovered from wound samples of patients submitted to minimally invasive surgery such as arthroscopy and laparoscopy. The isolates showed susceptibility to amikacin $\left(\mathrm{MIC}_{90}=4 \mu \mathrm{g} / \mathrm{mL}\right)$ and clarithromycin $\left(\mathrm{MIC}_{90}<1 \mu \mathrm{g} / \mathrm{mL}\right)$ but resistance to ciprofloxacin $\left(\mathrm{MIC}_{90}>16 \mu \mathrm{g} / \mathrm{mL}\right)$, doxycycline $\left(\mathrm{MIC}_{90}>32 \mu \mathrm{g} / \mathrm{mL}\right)$, sulfamethoxazole $\left(\mathrm{MIC}_{90}>128 \mu \mathrm{g} / \mathrm{mL}\right)$, and tobramycin $\left(\mathrm{MIC}_{90}=32 \mu \mathrm{g} / \mathrm{mL}\right)$, and intermediate profile to cefoxitin $\left(\mathrm{MIC}_{90}=64 \mu \mathrm{g} / \mathrm{mL}\right.$ ). Therefore, we suggest that the antimicrobial susceptibilities of any clinically significant RGM isolate should be performed.
\end{abstract}

\section{Introduction}

Infections by rapidly growing mycobacteria (RGM) are increasing in minimally invasively surgeries worldwide [1-3]. Mycobacterium massiliense has been isolated from pacemaker pocket infection, intramuscular injections, and post-video surgical infections [1, 2, 4-6]. Mycobacterium massiliense was validated as a separate species from the M. chelonae abscessus group in 2004 [4].

In Brazil, outbreaks caused by RGM have been reported since 1998. The former outbreaks occurred following laser in situ keratomileusis (surgery for myopia correction), mesotherapy sessions (intradermal injections) or breast implants. Likewise, in those outbreaks $M$. chelonae-abscessus group was the main pathogen found $[7,8]$. Recently, an epidemic of surgical-site infections was reported in seven different regions of Brazil, and surprisingly it was shown to be caused by a single clone of $M$. massiliense $[1,2,9,10]$.

RGM are intrinsically resistant to several antibiotic drugs reducing the number of active drugs to treat infections by these bacteria and therefore antimicrobial susceptibility testing have been shown to improve the clinical outcome [11-13]. For this reason, it is recommended that all clinically significant isolates should be tested against selected antimicrobial agents $[14,15]$.

The Clinical and Laboratory Standards Institute (CLSI) recommends the standard broth microdilution method for susceptibility testing of the Mycobacterium fortuitum group (M. fortuitum, M. peregrinum, and M. fortuitum third variant complex), Mycobacterium chelonae, and Mycobacterium abscessus. The method and guidelines for interpretation of results, on theoretical grounds, also should apply to Mycobacterium mucogenicum, Mycobacterium smegmatis group (M. smegmatis, M. goodii, and M. wolinskyi), and the clinically significant, pigmented RGM; however, data to support this are not currently available [16]. Being a recently classified RGM, M. massiliense susceptibility testing guidelines are needed, and susceptibility testing data from different settings will contribute for this goal. 
The aim of this work was to examine the in vitro susceptibilities of $M$. massiliense isolates from wound samples of patients with infection post-video surgeries such as arthroscopy and laparoscopy at seven private hospitals of Goiânia, Goiás, Brazil.

\section{Materials and Methods}

2.1. Mycobacterial Strains. The patients were from seven private hospitals in the city of Goiânia, State of Goiás, Brazil. Patient enrollment occurred between August 2005 and July 2007. Eighteen epidemic isolates of M. massiliense were included in this study, after patients signed consent agreement. The microorganisms were isolated from clinical samples of 18 patients that presented signs and symptoms of localized infection after minimally invasive surgery (arthroscopy or laparoscopy). M. massiliense strains were identified to the species level by PCR-restriction digestion of the hsp65 gene, pulsed-field gel electrophoresis (PFGE) comparisons, and rpoB partial gene sequencing [1]. Isolates were maintained on Lowenstein-Jensen slants prior to being tested and subcultured onto Mueller-Hinton plates at $35^{\circ} \mathrm{C}$ for 3 to 5 days. M. abscessus ATCC 19977 was used as a quality control strain.

2.2. Antimicrobial Agents and Microdilution Trays. Serial twofold dilutions of antimicrobial solutions were added to Mueller-Hinton broth to achieve final concentrations of antimicrobial agents (all from Sigma Aldrich, USA): amikacin (2 to $128 \mu \mathrm{g} / \mathrm{mL}$ ), cefoxitin (4 to $256 \mu \mathrm{g} / \mathrm{mL}$ ), ciprofloxacin $(0,25$ to $16 \mu \mathrm{g} / \mathrm{mL})$, clarithromycin (1 to $64 \mu \mathrm{g} / \mathrm{mL})$, doxycycline $(0,5$ to $32 \mu \mathrm{g} / \mathrm{mL})$, sulfamethoxazole (2 to $128 \mu \mathrm{g} / \mathrm{mL}$ ), and tobramycin $(0,5$ to $32 \mu \mathrm{g} / \mathrm{mL}$ ) and added across the 96-well plates.

2.3. Susceptibility Testing. Minimal inhibitory concentrations (MICs) of all tested drugs were determined by the broth microdilution method according to the guidelines described by the CLSI [16]. The final inoculum size was between $10^{4}$ and $10^{5} \mathrm{CFU} / \mathrm{mL}$. Inoculated plates were sealed inside plastic bags and incubated at $35^{\circ} \mathrm{C}$. All tests were performed in triplicate and the MICs were read at $72 \mathrm{~h}$. The susceptibility categories of all antimicrobial agents were determined according to the breakpoints recommended by CLSI [16].

\section{Results and Discussion}

Eighteen isolates of $M$. massiliense were recovered from wound samples of patients submitted to minimally invasive surgery such as arthroscopy $(n=14,77.8 \%)$ and laparoscopy $(n=4,22.2 \%)$.

All 18 strains tested were susceptible to amikacin ( $\mathrm{MIC}_{90}$ $=4 \mu \mathrm{g} / \mathrm{mL}$ ) and clarithromycin ( MIC $\left._{90}<1 \mu \mathrm{g} / \mathrm{mL}\right)$ but resistant to ciprofloxacin $\left(\mathrm{MIC}_{90}>16 \mu \mathrm{g} / \mathrm{mL}\right)$, doxycycline $\left(\mathrm{MIC}_{90}>32 \mu \mathrm{g} / \mathrm{mL}\right)$, sulfamethoxazole $\left(\mathrm{MIC}_{90}>\right.$ $128 \mu \mathrm{g} / \mathrm{mL})$, and tobramycin $\left(\mathrm{MIC}_{90}=32 \mu \mathrm{g} / \mathrm{mL}\right)$. All
Table 1: Antimicrobial susceptibility of 18 Mycobacterium massiliense strains recovered from wound infections after arthroscopic and laparoscopic surgeries during an outbreak in Goiânia, Goiás, Brazil.

\begin{tabular}{lccc}
\hline Antimicrobial & MIC $_{50}$ & MIC $_{90}$ & $\%$ \\
\hline Amikacin & $<2$ & 4 & $100(\mathrm{~S})$ \\
Cefoxitin & 32 & 64 & $100(\mathrm{I})$ \\
Ciprofloxacin & $>16$ & $>16$ & $100(\mathrm{R})$ \\
Clarithromycin & $<1$ & $<1$ & $100(\mathrm{~S})$ \\
Doxycycline & $>32$ & $>32$ & $100(\mathrm{R})$ \\
Sulfamethoxazole & $>128$ & $>128$ & $100(\mathrm{R})$ \\
Tobramycin & $>32$ & $>32$ & $100(\mathrm{R})$ \\
\hline
\end{tabular}

All results were determined by broth microdilution method; I: Intermediate; R: resistant; S: susceptible; $\mathrm{CIM}_{50}$ : the MIC capable of preventing the growth of $50 \%$ of the isolates; $\mathrm{MIC}_{90}$ : the MIC capable of preventing the growth of $90 \%$ of the isolates.

isolates had intermediate MICs for cefoxitin $\left(\mathrm{MIC}_{90}=\right.$ $64 \mu \mathrm{g} / \mathrm{mL})$. The results are summarized in Table 1 .

RGM are intrinsically resistant to the antibiotics used for tuberculosis treatment, consequently patients treatment with antituberculosis drugs for infection with those bacteria may become compromised [17]. Infections by RGM that have adequately and early identification of the microorganism will have a better result outcome [18]. Susceptibility testing is a powerful tool in order to point for the use of the most effective drug and consequently enhancing the treatment success rate.

Our susceptibility profile results corroborate with those of Adékambi et al. [4], except for the elevated MIC values encountered for doxycycline. In regard to this particular drug, other groups have reported similar findings $[2,6,9]$. This finding strengthen the idea that doxycycline should not be used as a differentiation marker between $M$. massiliense and $M$. abscessus [4].

According to CLSI [16], results for tobramycin should not be reported for the M. fortuitum or M. abscessus groups, as the treatment with this drug will only be superior to amikacin in infections caused by $M$. chelonae. No susceptibility testing recomendation is avaliable for $M$. massiliense yet but, according to our data, this drug should not be used, as all isolates were resistant to tobramycin. We have encountered $100 \%$ of resistance among the strains tested for sulfamethoxazole, similarly to the susceptibility reported for M. chelonae e M. abscessus [16].

Clarithromycin has been indicated as the first-line drug of choice for the treatment of infections caused by RGM $[14,19]$ and is appropriate for M. massiliense as well, as all of the strains tested for this drug in our study were susceptible. Koh et al. (2010) compared treatment outcomes of patients infected with either M. abscessus or $M$. massiliense and concluded that treatment response rates to combination antibiotic therapy including clarithromycin were much higher in patients with $M$. massiliense than in those with $M$. abscessus lung disease [20].

Acquired resistance to clarithromycin has been reported with a rate of $2.3 \%$ in patients receiving monotherapy [21] 
and one death have been reported due to infection with a resistant strain [22]. A previous monotherapy trial of clarithromycin for cutaneous disease caused by $M$. chelonae in immunosuppressed patients (all patients were on corticosteroids) resulted in acquired resistance among isolates from 1 of $10(10 \%)$ patients with disseminated disease and none of $4(0 \%)$ patients with localized disease [23]. Because of the risks relative to resistance development, it has been recommended the association of a second drug in the treatment for infections with these bacteria. Amikacin seems a good candidate, as in our study all strains were susceptible to this drug.

Another drug that is associated with clarithromycin to treat infections with RGM is cefoxitin [24], however our results showed that $M$. massiliense isolates presented an intermediary susceptibility to this drug. The different profile of susceptibilities found in our study and others stress the need for the proper RGM identification followed by a drug susceptibility screening in order to provide the most appropriate antibiotic treatment.

The treatment of serious infections with RGM is a problem and limited by the small number of available drugs with activity at clinically achievable levels in tissue or/and blood. Each species and strain must be individually evaluated, and it is advisable always to perform in vitro sensitivity tests before using the drug for human therapy [25].

\section{Conclusions}

In conclusion, this study found that the MICs were higher for M. massiliense when tested cefoxitin, ciprofloxacin, doxycycline, sulfamethoxazole and tobramycin. Therefore, amikacin and clarithromycin were active against M. massiliense strains isolated in our study.

\section{Acknowledgments}

The authors received financial support from OPAS/OMSBrazil and ANVISA (Termo de Cooperação 37). A. Kipnis and A. P. Junqueira-Kipnis received fellowships from CNPqBrasil.

\section{References}

[1] A. M. Cardoso, E. Martins de Sousa, C. Viana-Niero et al., "Emergence of nosocomial Mycobacterium massiliense infection in Goiás, Brazil," Microbes and Infection, vol. 10, no. 14-15, pp. 1552-1557, 2008.

[2] R. S. Duarte, M. C. S. Lourenço, L. D. S. Fonseca et al., "Epidemic of postsurgical infections caused by Mycobacterium massiliense," Journal of Clinical Microbiology, vol. 47, no. 7, pp. 2149-2155, 2009.

[3] A. Carbonne, F. Brossier, I. Arnaud et al., "Outbreak of nontuberculous mycobacterial subcutaneous infections related to multiple mesotherapy injections," Journal of Clinical Microbiology, vol. 47, no. 6, pp. 1961-1964, 2009.

[4] T. Adékambi, M. Reynaud-Gaubert, G. Greub et al., "Amoebal coculture of "Mycobacterium massiliense" sp. nov. from the sputum of a patient with hemoptoic pneumonia," Journal of Clinical Microbiology, vol. 42, no. 12, pp. 5493-5501, 2004.

[5] H. Y. Kim, Y. J. Yun, C. G. Park et al., "Outbreak of Mycobacterium massiliense infection associated with intramuscular injections," Journal of Clinical Microbiology, vol. 45, no. 9, pp. 3127-3130, 2007.

[6] K. E. Simmon, J. I. Pounder, J. N. Greene et al., "Identification of an emerging pathogen, Mycobacterium massiliense, by rpoB sequencing of clinical isolates collected in the United States," Journal of Clinical Microbiology, vol. 45, no. 6, pp. 1978-1980, 2007.

[7] D. Freitas, L. Alvarenga, J. Sampaio et al., "An outbreak of Mycobacterium chelonae infection after LASIK," Ophthalmology, vol. 110, no. 2, pp. 276-285, 2003.

[8] J. L. M. Sampaio, E. Chimara, L. Ferrazoli et al., "Application of four molecular typing methods for analysis of Mycobacterium fortuitum group strains causing post-mammaplasty infections," Clinical Microbiology and Infection, vol. 12, no. 2, pp. 142-149, 2006.

[9] C. Viana-Niero, K. V. B. Lima, M. L. Lopes et al., "Molecular characterization of Mycobacterium massiliense and Mycobacterium bolletii in isolates collected from outbreaks of infections after laparoscopic surgeries and cosmetic procedures," Journal of Clinical Microbiology, vol. 46, no. 3, pp. 850-855, 2008.

[10] S. C. Leão, C. Viana-Niero, C. K. Matsumoto et al., "Epidemic of surgical-site infections by a single clone of rapidly growing Mycobacteria in Brazil," Future Microbiology, vol. 5, no. 6, pp. 971-980, 2010.

[11] J. M. Swenson, R. J. Wallace Jr., V. A. Silcox, and C. Thornsberry, "Antimicrobial susceptibility of five subgroups of Mycobacterium fortuitum and Mycobacterium chelonae," Antimicrobial Agents and Chemotherapy, vol. 28, no. 6, pp. 807-811, 1985.

[12] R. J. Wallace Jr., J. M. Swenson, V. A. Silcox, and M. G. Bullen, "Treatment of nonpulmonary infections due to Mycobacterium fortuitum and Mycobacterium chelonei on the basis of in vitro susceptibilities," Journal of Infectious Diseases, vol. 152, no. 3, pp. 500-514, 1985.

[13] S. Park, S. Kim, E. M. Park et al., "In vitro antimicrobial susceptibility of Mycobacterium abscessus in Korea," Journal of Korean Medical Science, vol. 23, no. 1, pp. 49-52, 2008.

[14] D. E. Griffith, T. Aksamit, B. A. Brown-Elliott et al., "An official ATS/IDSA statement: diagnosis, treatment, and prevention of nontuberculous mycobacterial diseases," American Journal of Respiratory and Critical Care Medicine, vol. 175, no. 4, pp. 367416, 2007.

[15] C. L. Daley and D. E. Griffith, "Pulmonary disease caused by rapidly growing mycobacteria," Clinics in Chest Medicine, vol. 23, no. 3, pp. 623-632, 2002.

[16] CLSI, "Susceptibility Testing of Mycobacteria, Nocardiae, and Other Aerobic Actinomycetes. Approved Standard," CLSI Document M24-A, vol. 23, no. 18, pp. 25-32, 2003.

[17] A. J. Eid, E. F. Berbari, I. G. Sia, N. L. Wengenack, D. R. Osmon, and R. R. Razonable, "Prosthetic joint infection due to rapidly growing mycobacteria: report of 8 cases and review of the literature," Clinical Infectious Diseases, vol. 45, no. 6, pp. 687694, 2007.

[18] M. Pring and D. G. Eckhoff, "Mycobacterium chelonae infection following a total knee arthroplasty," Journal of Arthroplasty, vol. 11, no. 1, pp. 115-116, 1996.

[19] B. A. Brown-Elliott and R. J. Wallace Jr., "Clinical and taxonomic status of pathogenic nonpigmented or late-pigmenting rapidly growing mycobacteria," Clinical Microbiology Reviews, vol. 15, no. 4, pp. 716-746, 2002. 
[20] W. J. Koh, K. Jeon, and N. Y. Lee, "Clinical significance of differentiation of Mycobacterium massiliense from Mycobacterium abscessus," American Journal of Respiratory and Critical Care Medicine. In press.

[21] R. J. Wallace Jr., A. Meier, B. A. Brown et al., "Genetic basis for clarithromycin resistance among isolates of Mycobacterium chelonae and Mycobacterium abscessus," Antimicrobial Agents and Chemotherapy, vol. 40, no. 7, pp. 1676-1681, 1996.

[22] M. Sanguinetti, F. Ardito, E. Fiscarelli et al., "Fatal pulmonary infection due to multidrug-resistant Mycobacterium abscessus in a patient with cystic fibrosis," Journal of Clinical Microbiology, vol. 39, no. 2, pp. 816-819, 2001.

[23] R. J. Wallace, D. Tanner, P. J. Brennan, and B. A. Brown, "Clinical trial of clarithromycin for cutaneous (disseminated) infection due to Mycobacterium chelonae," Annals of Internal Medicine, vol. 119, no. 6, pp. 482-486, 1993.

[24] A. Scholze, C. Loddenkemper, M. Grünbaum, I. Moosmayer, G. Offermann, and M. Tepel, "Cutaneous Mycobacterium abscessus infection after kidney transplantation," Nephrology Dialysis Transplantation, vol. 20, no. 8, pp. 1764-1765, 2005.

[25] C. Cavusoglu, I. Soyler, and P. Akinci, "Activities of Linezolid against nontuberculous mycobacteria," New Microbiologica, vol. 30, no. 4, pp. 411-414, 2007. 


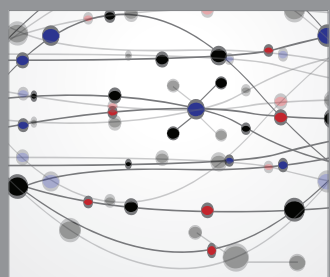

The Scientific World Journal
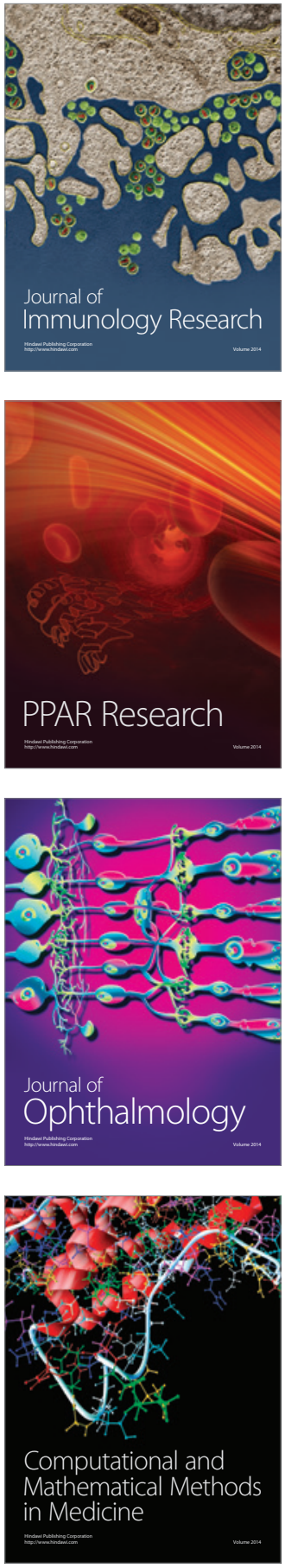

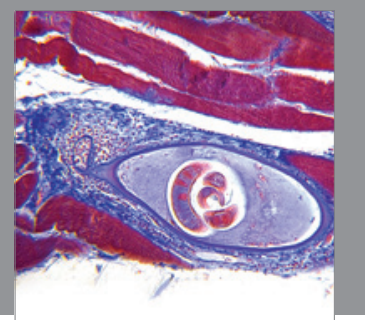

Gastroenterology

Research and Practice
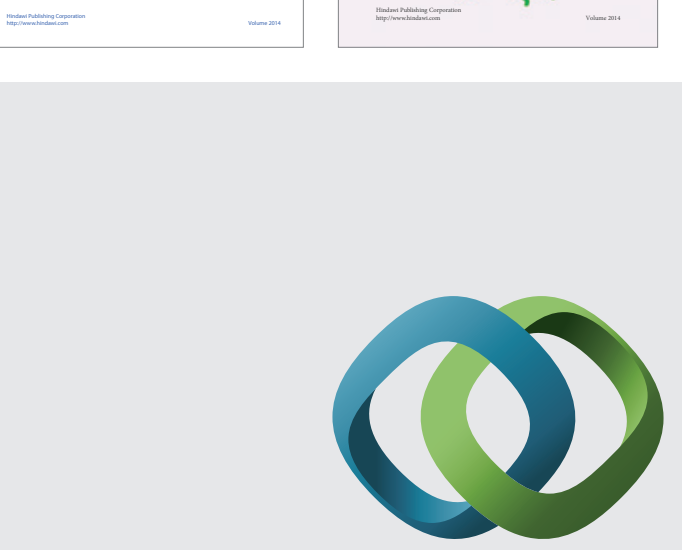

\section{Hindawi}

Submit your manuscripts at

http://www.hindawi.com
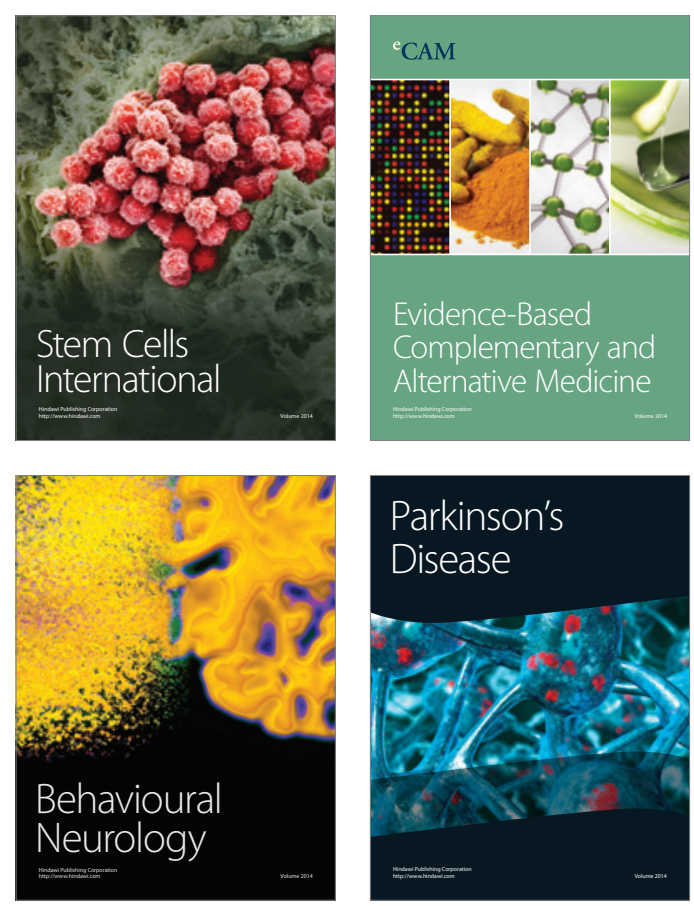

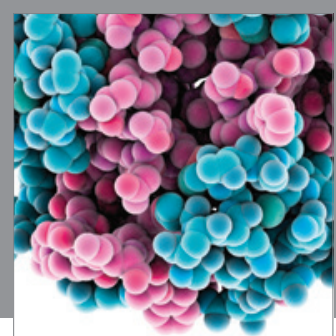

Journal of
Diabetes Research

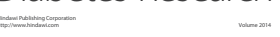

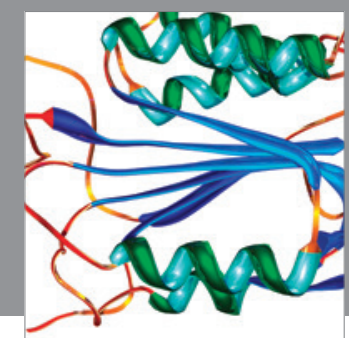

Disease Markers
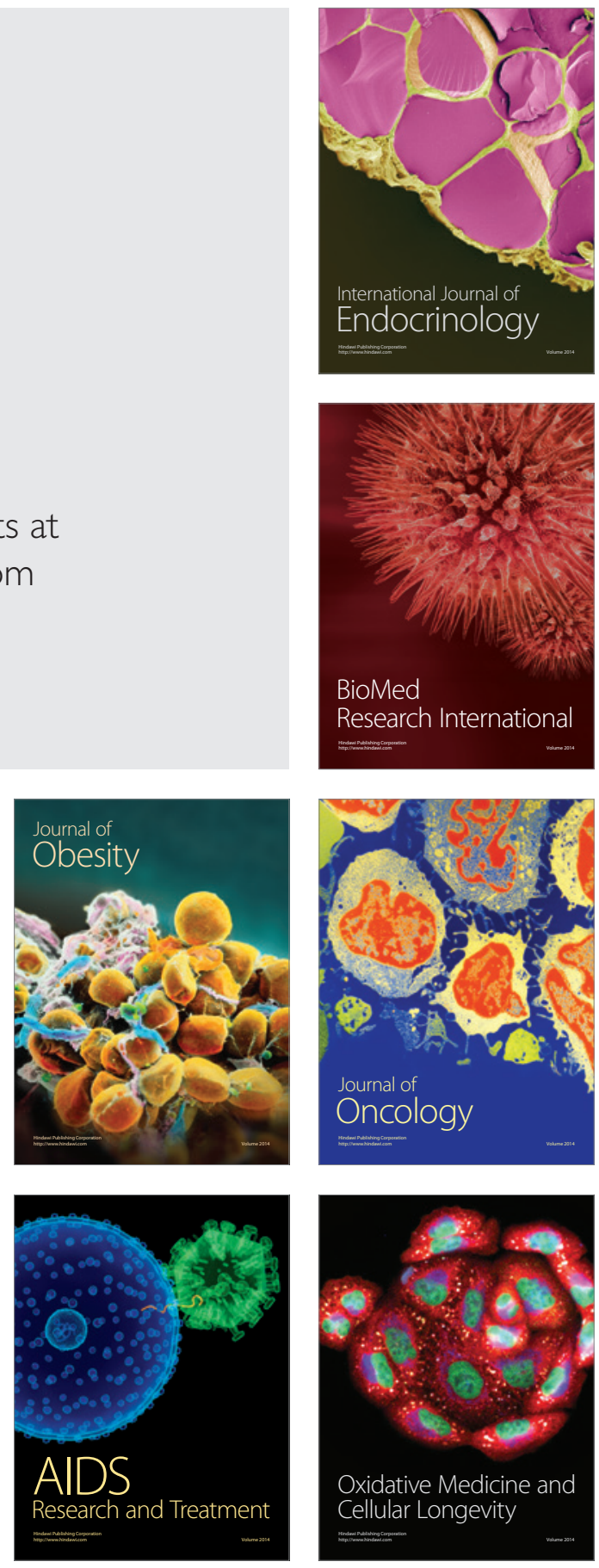